\title{
Cutaneous Cholesterol Embolization
}

\author{
Gulden Avci ${ }^{1}$, Tayfun Akoz ${ }^{2}$, Aylin Ege Gul ${ }^{3}$
}

1. Department of Plastic and Reconstructive Surgery, Canakkale Onsekiz Mart University, Canakkale, Turkey

2. Department of Plastic and Reconstructive Surgery, Dr. Lutfi Kirdar Kartal Education and Research Hospital, Istanbul, Turkey

3. Department of Pathology, Dr. Lutfi Kirdar Kartal Education and Research Hospital, Istanbul, Turkey

Corresponding author:

\section{Dr Gulden Avci}

Canakkale Onsekiz Mart University

Faculty of Medicine,

Department of Plastic and

Reconstructive Surgery

Canakkale, Turkey

E-mail:drguldenavci@hotmail.com

\section{Key words:}

cholesterol embolization, renal insufficiency, skin necrosis, ulcer

\begin{abstract}
Background: Cutaneous cholesterol embolization syndrome occurs as a result of cholesterol embolization from atherosclerotic plaques lining the walls of arteries and arterioles. It can occur sporadically but is more commonly associated with iatrogenic manipulation via invasive vascular procedures or therapies (anticoagulation or thrombolytics) The three most common organ systems afflicted by cholesterol emboli include the kidneys, gastrointestinal system, and skin.
\end{abstract}

Main Observations: We present two patients with cutaneous cholesterol embolization. A 74-year-old with no prior disease or trauma was referred to the hospital with the acute chest pain. After 24-48 he developed livedo reticularis, followed by necrotic areas like bilaterally on hips and in the lumbar region. The second patient, 69-year-old man with a history of hyperglycemia, hyperlipidaemia, and hypertension was referred with weight loss, bilateral livedo reticularis, severe pain of lower extremities and impaired renal function. The toes first became cyanotic and than skin necrosis developed. Skin biopsy revealed presence of cholesterol clefts in the lumina of small arteries and arterioles. In both patients the necrotic skin and subcutaneous tissue had been debrided. Daily dressing was applied and tissue defects underwent secondary healing.

Conclusion: The diagnosis of cholesterol embolization relies on clinical and histologic examination. Both, diagnosis and treatment needs a multidisciplinary approach, especially in cases of multi-organ involvement.

\section{Introduction}

Cholesterol crystal embolization syndrome (CES) is a systemic disorder due to embolization of atheromatous material from ulcerated atherosclerotic in the major arteries. Panum first described atheromatous embolism in 1862. ${ }^{1}$ However, little was known about this syndrome until Flory described the basic pathophysiology of CES in an autopsy report in 1946. The mortality of CES was reported to be $81 \%$. This may be the result of the diverse clinical presentation and the poor clinical recognition disorder. $^{2}$

We present 2 patients with CES each associated a different risk factor.

\section{Case reports}

\section{Patient 1}

A 74-year-old man was referred with the acute chest pain to the department of internal medicine. He had S-T elevation on the admission electrocardiogram consistent with an acute myocardial infarction. He had no prior any disease or trauma. He was started on streptokinase (1.5 million U/1 hour), intravenous heparin and oral aspirin. Approximately 24-48 hours later he had bilateral livedo reticularis in inguinal regions, hips and lumbal area. He developed belt-shaped necrotic lesion bilateral on hips and lumbar regions (Fig. 1A-C). A preliminary diagnosis of "necrotizing vasculitis" was considered. The patient had good peripheral pulses and a normal blood pressure. The 
white blood count was normal, total blood chemistry was within normal range.

An incisional biopsy was performed in the area of livedo reticularis just proximal to the area of necrosis. Histology revealed cholesterol clefts within arteries with no evidence of vasculitis (Fig. 1D). Anticoagulant therapy was discontinued. The necrotic skin and subcutaneous tissue had been debrided. Daily dressing was applied and tissue defects underwent secondary healing. There was no further progression of cutaneous lesions or evidence of systemic involvement. Wounds gradually healed.

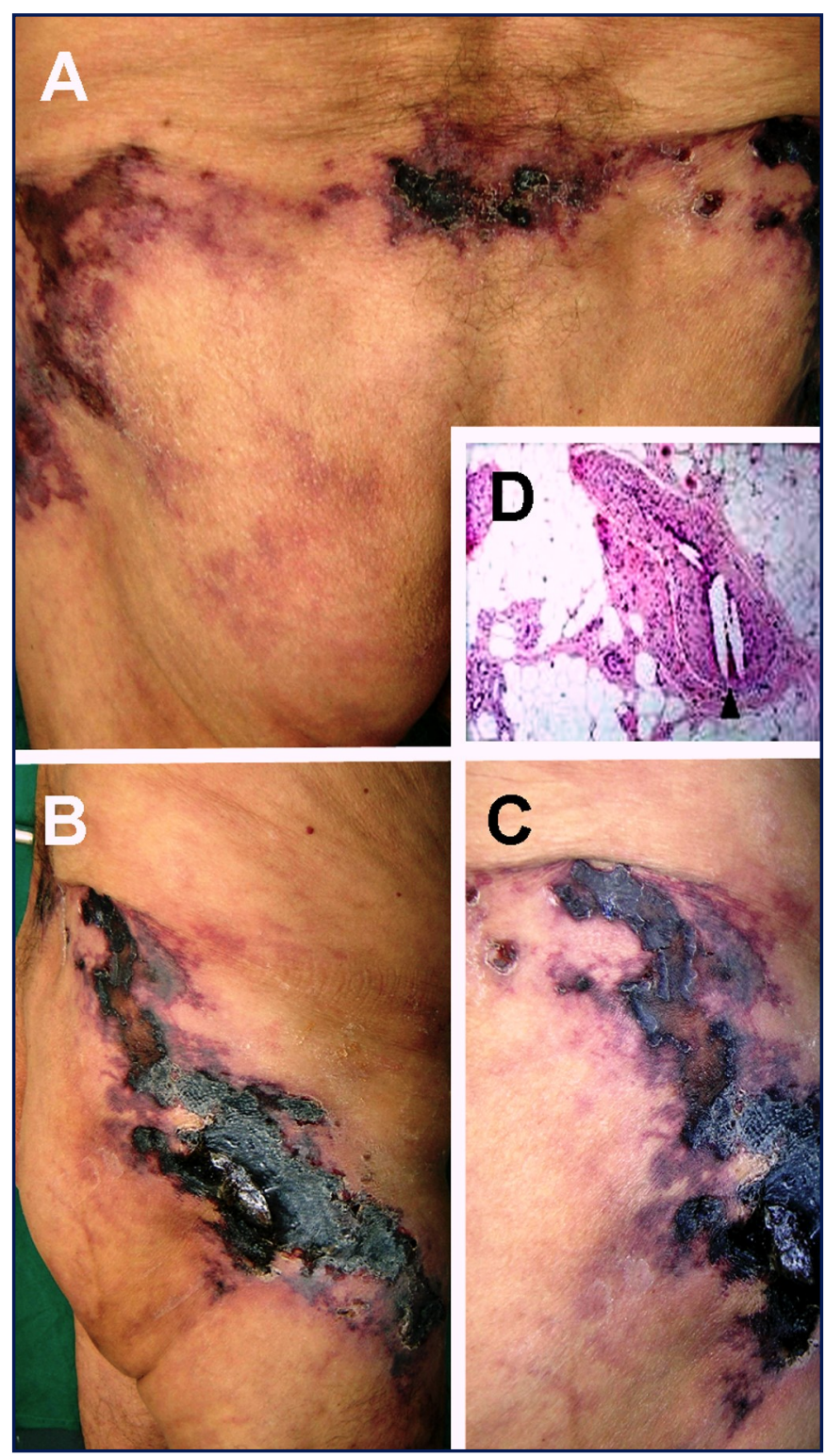

Figure 1

Patient 1 with cutaneous cholesterol embolization. Necrosis on the hips $(A)$ and lumbar region $(B, C)$. Skin histology showing cholesterol clefts in the lumen of a vessel (hematoxyline eosine, $x 40,(D))$.

\section{Patient 2}

A 69-year-old man was referred to the cardiovascular surgery unit with weight loss, bilateral livedo reticularis and extremely pain of lower extremities. On presentation, his peripheral pulses were normal. Erythrocyte sedimentation rate was elevated at $112 \mathrm{~mm}$ in the first hour. The leukocyte count was 10300 cells/mcL with $11 \%$ eosinophils. He had a history of hyperglycemia, hyperlipidaemia, and hypertension. The initial clinical diagnosis was a vasculitis. During hospitalization, he developed renal insufficiency with serum creatinine concentration up to 5.6 $\mathrm{mg} / \mathrm{dl}$. Prednisone therapy was initiated. Shortly thereafter the first right toe and the fifth left toe became cyanotic and necrosis developed in the plantar regions (Fig. 2A). Because of steroid therapy, it was decided to not perform surgery, remain conservative and observe the progression of the lesions, while the patient recovered from acute renal failure. An incisional biopsy was performed just proximal to an area of necrosis within an area of livedo reticularis on the right toe. Skin biopsy revealed presence of cholesterol clefts in the lumina of small arteries and arterioles (Fig. 2B). During the one and half month long treatment renal function improved and steroid dose was tapered. All the necrotic tissues were debrided and some of the defects were primarily sutured. Remaining tissue defects underwent secondary healing. We performed daily wound care. Wound healing was completed without any complications.

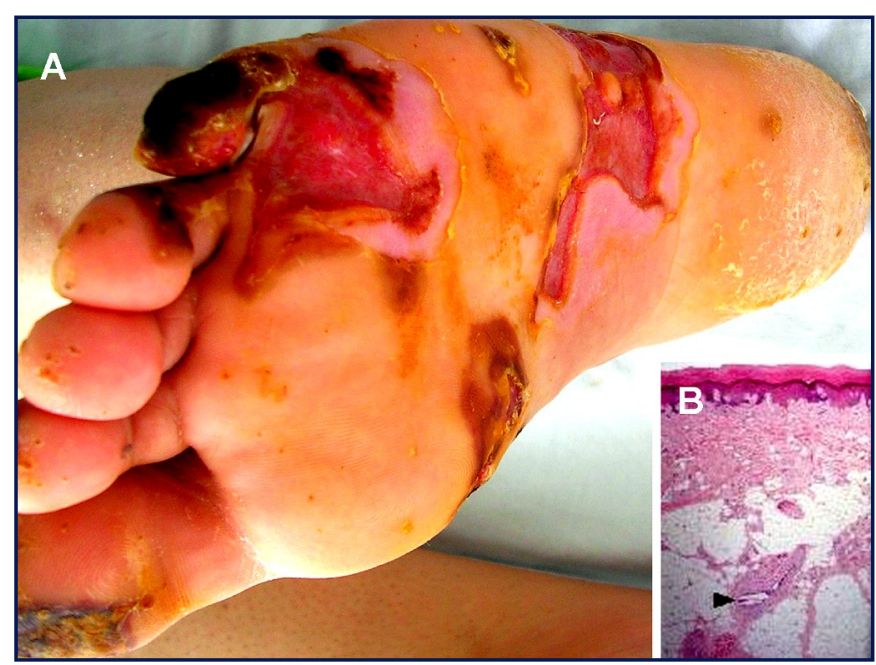

Figure 2

Patient 2 with cutaneous cholesterol embolization. Necrotic lesions on the foot (A). Skin biopsy showes cholesterol clefts in the lumen of a vessel (hematoxyline eosine, $x 10,(B)$ ).

\section{Discussion}

Cholesterol emboli are one of the many complications of atherosclerosis. Atheromatous plaques remain stable for many years. But they enlarge over time, becoming friable and prone to erosion in later years. Numerous factors are thought to contribute to this unstable nature and to the subsequent rupture or erosion. These factors include inflammation, sheering forces from blood flow (particularly in hypertension), hemorrhage within the plaque, aneurysm formation, infection, and calcification, mechanical 
manipulation during surgery or vascular procedures and anticoagulation. When cholesterol emboli are released into the bloodstream, they travel distally until they lodge in small arterioles that characteristically range from $100 \mu \mathrm{m}$ to $200 \mu \mathrm{m}$ in size. ${ }^{3}$ With the aorta being the leading site of atherosclerosis, the downstream sites of kidneys, viscera and lower extremities show the highest frequency of involvement. Pedal pulses are generally normal, absent in $19 \%$ and unilaterally absent in $2 \%$ of patients. ${ }^{4}$

CES affects predominantly elderly males (mean age 69.2 years old), with a male to female ratio of $3.4: 1$. Patients have a history of hypertension $(61 \%)$, atherosclerotic disease $(44 \%)$, or aortic aneurysm (8.3\%). ${ }^{2}$ A history of myocardial infarction was reported in $23 \%$ of the cases. ${ }^{2}$ Spontaneous cholesterol embolism without any predisposing factor was also described. Invasive angiography is one of the most common factors leading to the development of cholesterol embolism (10\%). A prior coronary angiogram was reported in $4 \%$ of cases. ${ }^{3,4}$ Cholesterol embolization also has been associated with major vessel surgery, angioplasty intra-aortic balloon pump placement, anticoagulation, thrombolytic therapy and cardiopulmonary resuscitation. 5 Thrombolytic therapy and anticoagulants appear to precipitate cholesterol emboli by dissolving protective thrombi and fibrin deposits coating an atheromatous plaque, permitting the release of cholesterol. ${ }^{6}$ In addition, CES may occur spontaneously. Diabetes, hypertension and diffuse vascular disease are major risk factors for both iatrogenic and spontaneous CES. Among them, diabetes mellitus, often in the context of the "metabolic syndrome", is probably the most important risk element. ${ }^{7}$

CES is a complex disorder, which may be suspected based on certain clinical and laboratory abnormalities. The major clinical signs and symptoms are related to ischemic lesions in the tissue with higher vascularization. However, there is no single tissue spared by CES. ${ }^{6}$ Almost every organ can be affected in this syndrome, but the three areas of greatest involvement are the skin, kidneys and digestive system. ${ }^{2}$ Cutaneous changes are seen in $69.4 \%$ of patients throughout the course of CES. ${ }^{4}$

Dermatologic manifestations of cholesterol emboli are varied. Livedo reticularis, and acrocyanosis of the lower extremities are the most commonly described cutaneous findings. Livedo reticularis consists of blue-red mottling of the skin in a net-like pattern usually seen over the feet, legs, buttocks and lumbar regions. ${ }^{8}$ The cutaneous findings are often bilateral and limited to the lower extremities. Peripheral pulses are usually normal. Purple or blue toe syndromes are consequences of cholesterol embolism. ${ }^{9}$ Other dermatologic manifestations of cholesterol embolism consists of ulceration of the legs and feet, purpura and nodular lesions, suggestive of polyarteritis nodosa, and penile and scrotal necrosis. ${ }^{3}$

The multiple organ involvement of CES often mimics a systemic vasculitis or a connective tissue disorder. The differential diagnosis in patients with CES often includes both autoimmune or pauci-immune vasculitides and septic embolism. Other systemic diseases such as endocarditis and polimyositis may also be considered in the differential diagnosis. CES is most probably underdiagnosed. This occurs because: 1) symptoms are often atypical, especially in patients with prominent visceral disease 2) symptoms are frequently not reported by the patients 3) biopsies are often not performed or diagnostic features are not seen, perhaps because sectioning through the block is not routinely ordered when initial sections are diagnostic. ${ }^{1}$

Treatment options for CES are limited and nowadays there is no universally accepted pharmacologic treatment. ${ }^{10}$ Some authors indicate the need of cessation of anticoagulant therapy with suspicion of the diagnosis. ${ }^{11}$ Corticosteroid treatment is controversial. Systemic and multidisciplinary approach should be applied on case to case basis. In the literature, we couldn't find a suggested standard surgical treatment of skin lesions. There will always be a challenging and demanding defect requiring the mastery of a wide range of reconstructive options.

\section{References}

1. Pennington $\mathrm{M}$, Yeager J, Skelton $\mathrm{H}$, Smith KJ. Cholesterol embolization syndrome: cutaneous histopathological features and the variable onset of symptoms in patients with different risk factors. B J Dermatol. 2002; 146: 511-517.

2. Funabiki $K$, Masuoka H, Shimizu H, Emi Y, Mori T, Ito M, Nakano T. Cholesterol crystal embolization (CCE) after cardiac catheterization: a case report and a review of 36 cases in the Japanese literature. Review. Jpn Heart J. 2003; 44: 767-774.

3. Falanga V, Fine MJ, Kapoor WN. The cutaneous manifestations of cholesterol crystal embolization. Arch Dermatol. 1986; 122: 1194-1198.

4. Fine MJ, Kapoor W, Falanga V. Cholesterol crystal embolization: a review of 221 cases in the English literature. Angiology. 1987; 38: 769-784.

5. Piccoli GB, Sargiotto A, Burdese M, Colla L, Bilucaglia D, Magnano A, Consiglio V, Piccoli G, Picciotto G. Cholesterol emboli syndrome in type 2 diabetes: the disease history of a case evaluated with renal scintigraphy. Rev Diabet Stud. 2005; 2: 92-96.

6. Hitti WA, Wali RK, Weinman EJ, Drachenberg C, Briglia A. Cholesterol embolization syndrome induced by thrombolytic therapy. Am J Cardiovasc Drugs. 2008; 8: 27-34.

7. Rivera F, Vazmediano C, Gonzalez-Lopez L, Carrano A, Blanco J. Subacute renal failure in diabetic nephropathy due to endocapillary glomerulonephritis and cholesterol embolization. J Nephrol. 2008; 21: 615-620.

8. Fukumoto Y, Tsutsui H, Tsuchihashi M, Masumoto A, Takeshita A. Cholesterol Embolism Study (CHEST) Investigators. The incidence and risk factors of cholesterol embolization syndrome, a complication of cardiac catheterization: a prospective study. J Am Coll Cardiol. 2003; 42: 211-216.

9. Borrego L, Gil R, Mazuecos A, Ruiz R, López JL, Iglesias L. Cholesterol embolism to the skin. Clin Exp Dermatol. 1992; 17: 424-426.

10. Erdim M, Tezel E, Biskin N. A case of skin necrosis as a result of cholesterol crystal embolisation. J Plast Reconstr Aesthet Surg. 2006; 59: 429-432.

11. Lane JE, Lane TN, Shams M, Lane CE. Cutaneous cholesterol embolization. J Am Acad Dermatol. 2009; 60: 711-713. 